\title{
Resistance to interference in complex negative patterning
}

\author{
DOUGLAS A. WILLIAMS, JENNIFER D. GAWEL, and DALLAS S. REIMER \\ University of Winnipeg, Winnipeg, Manitoba, Canada \\ and \\ RICK MEHTA \\ Acadia University, Wolfville, Nova Scotia, Canada
}

\begin{abstract}
Resistance to interference was examined in rats that received a complex negative patterning discrimination in which XA and XB were followed by food reinforcement and XAB was not. Retention of the discrimination was evident after separate reinforcement of both A and B (Experiment 3), but not after reinforcement of either $\mathrm{AB}$ (Experiments 1 and 3) or XAB (Experiments 2 and 3). These data suggest that complex negative patterning discriminations are acquired configurally and that the relative similarity of the original discrimination and subsequent interference trials dictates the final degree of retention observed.
\end{abstract}

Negative patterning is said to have occurred when an animal learns to respond to $\mathrm{A}$ and $\mathrm{B}$ individually, but not to the $\mathrm{AB}$ combination (i.e., $\mathrm{A}+, \mathrm{B}+, \mathrm{AB}-$; where "+" and "-" stand for reinforced and unreinforced trials, respectively). Such learning is of considerable interest for theory development, because it suggests that configural representations play an integral role in the association formation process (Woodbury, 1943). Distinctive conditioned stimuli (CSs) appear to combine to form representations of undifferentiated wholes, which may then act as stimuli in their own right, entering into association with the unconditioned stimulus (US). On the other hand, the summed excitatory associative strength of A and B can never be less than that of either element alone; thus, it is not obvious how the discrimination might be solved if the compound were merely the sum of its parts (Rescorla, 1972). Indeed, it is widely accepted that negative patterning is an anathema to purely elemental theories, and it may simply be impossible to formulate a comprehensive account of conditioning without assuming that compound stimuli evoke special representations upon the conjoint occurrence of several CSs. Some examples of configural mechanisms are unique cues evoked when two or more stimuli are simultaneously present (Rescorla, 1972, 1973), new internal representations that develop over the course of conditioning in multilayered networks when the individual CSs are poor predictors of the US (Kehoe, 1988; Schmajuk \& DiCarlo, 1992), or special units that underlie conditioning in all its varied forms (Pearce, 1987, 1994, 2002).

This research was supported by a grant from the Natural Sciences and Engineering Research Council of Canada. Correspondence concerning this article should be addressed to D. A. Williams, Psychology Department, University of Winnipeg, Winnipeg, MB, R3B 2E9 Canada (e-mail: williams@uwinnipeg.ca).
Attempts to reconcile a purely elemental theory with negative patterning have included appeals to trial-related background stimuli. According to this account, conditioning trials contain shared elements, in addition to the distinctive stimuli under the experimenter's control. Recognition of these shared elements, labeled X collectively, produces a more complex discrimination that has a linear solution, $\mathrm{XA}+, \mathrm{XB}+, \mathrm{XAB}-$. For example, the Rescorla-Wagner (1972) theory assumes that individual CSs gain or lose associative strength $(V)$ until the animal's expectations are consistent with the US obtained on a given trial. Trial-bytrial error correction occurs when the reinforcer obtained, $\lambda$, differs from the algebraic sum of all the CSs present, $V_{\text {all }}$, as formalized in the equation $\Delta V=\alpha \beta\left(\lambda-V_{\text {all }}\right)$. Typical values for $\lambda$ for the purposes of simulation are 1 for reinforced trials and 0 for unreinforced trials. The quantities $\alpha$ and $\beta$ are rate-learning parameters for individual CSs and the US, respectively, which limit the magnitude of associative change on a single trial.

Applied to complex negative patterning, the RescorlaWagner (1972) theory expects X to receive increments in associative strength, gaining more positive strength than its competitors $\mathrm{A}$ and $\mathrm{B}$, because it is reinforced on both $\mathrm{XA}+$ and $\mathrm{XB}+$ trials. All three elements receive decrements in associative strength on $\mathrm{XAB}$ - trials, which leaves X excitatory and A and B inhibitory. Assuming equal salience, the theory predicts the following terminal values for $V_{\text {all }}$, as expressed in this set of linear equations:

$$
\begin{aligned}
& \text { Trial XA: } V \mathrm{X}+V \mathrm{~A}=1 \lambda, \\
& \text { Trial } \mathrm{XB}: V \mathrm{X}+V \mathrm{~B}=1 \lambda,
\end{aligned}
$$

and

Trial XAB: $V \mathrm{X}+V \mathrm{~A}+V \mathrm{~B}=0 \lambda$.

Interactions between the components $\mathrm{A}, \mathrm{B}$, and $\mathrm{X}$ are expected to result in terminal strengths of $-1 \lambda,-1 \lambda$, and $2 \lambda$, 
respectively. Responding is expected to approach $1 \lambda$ when $\mathrm{X}$ is accompanied by either $\mathrm{A}$ or $\mathrm{B}(\mathrm{XA}=\mathrm{XB}=2 \lambda+$ $-1 \lambda=1 \lambda)$ and to approach $0 \lambda$ when both $\mathrm{A}$ and $\mathrm{B}$ are present $(\mathrm{XAB}=2 \lambda+-1 \lambda+-1 \lambda=0 \lambda)$. This potential reconciliation of elemental theory with negative patterning is encouraged by recent demonstrations that $X$ derives superexcitatory properties after $\mathrm{XA}+, \mathrm{XB}+, \mathrm{XAB}-$ discrimination learning (Williams et al., 2002), when $X$ is a discrete CS or a static context.

One serious problem for this particular account is that the introduction of a discrete $\mathrm{X}$ on all the trials of a negative patterning discrimination has been shown to retard rather than facilitate acquisition of negative patterning discriminations (Pearce \& Redhead, 1993). Indeed, it is just this problem that caused Rescorla (1972) to suggest that unique cues might be activated when elements are simultaneously presented. Other aspects of the experiments of Williams et al. (2002) are also not readily explained by this particular application of the Rescorla-Wagner (1972) model. Williams et al. (2002) reported that A and B did not pass summation tests of conditioned inhibition after complex negative patterning, as would be expected according to the Rescorla-Wagner model. In another experiment, they reported that a complex negative patterning discrimination was retained after separate reinforcement of $A$ and B. Because A, B, and X were demonstrably excitatory after separate reinforcement, the associative strengths of the three CSs should have positively summated, and the discrimination should have been reversed (i.e., $\mathrm{XA}=$ $\mathrm{XB}<\mathrm{XAB}$ ). Finally, in another series of experiments, $\mathrm{X}$ was found to retain its superexcitatory properties when new trials were added to the discrimination that made it nonlinearly solvable (Williams, Mehta, \& Dumont, 2004). Taken together, these findings pose a major challenge for the elemental theory of Rescorla and Wagner.

On the other hand, all of the preceding data can be explained by an alternative conceptualization of how stimuli are represented in compound. According to configural theory (Pearce, 1987), the entire stimulus complex as a whole enters into association with the US. Discrimination learning occurs when the animal responds to one stimulus complex, and not to another that might otherwise evoke responding through similarity-based generalization. Levels of similarity are determined by the product of the proportion of common elements in the various complexes. For example, if one element of a two-element complex were also present in a three-element complex, the stimulus complexes would be one-sixth similar $(.5 \times .33=$ .167). As with the Rescorla-Wagner (1972) model, it is possible to implement configural theory as a set of linear equations, to quickly determine asymptotic values. In this case, the uppercase letters refer to stimulus complexes as a whole, and the values in parentheses refer to the similarities of related stimulus complexes to the complex being considered.

$$
\begin{aligned}
& \text { Trial XA: } V_{\mathrm{XA}}+(.25) V_{\mathrm{XB}}+(.67) V_{\mathrm{XAB}}=1 \lambda, \\
& \text { Trial XB: }(.25) V_{\mathrm{XA}}+V_{\mathrm{XB}}+(.67) V_{\mathrm{XAB}}=1 \lambda,
\end{aligned}
$$

and

$$
\text { Trial XAB: (.67) } V_{\mathrm{XA}}+(.67) V_{\mathrm{XB}}+V_{\mathrm{XAB}}=0 \lambda \text {. }
$$

Solving this last set of linear equations reveals strengths of $2.77 \lambda$ for $V_{\mathrm{XA}}, 2.77 \lambda$ for $V_{\mathrm{XB}}$, and $-3.69 \lambda$ for $V_{\mathrm{XAB}}$ at asymptote. High levels of excitatory generalization from each of XA and XB and lower levels of inhibitory generalization from $\mathrm{XAB}$ form the basis of the explanation of why $\mathrm{X}$ derives superexcitatory properties in test [Trial $\left.\mathrm{X}=(.5) V_{\mathrm{XA}}+(.5) V_{\mathrm{XB}}+(.33) V_{\mathrm{XAB}}=1.54 \lambda\right]$.

Of special relevance for the present experiments is the added prediction that reinforcement of $\mathrm{A}$ and $\mathrm{B}$ after discrimination learning should not undermine retention of the original discrimination. According to configural theory, the stimulus complexes A and B would initially be novel and should evoke minor levels of responding through generalization. In particular, A is expected to receive half of the excitatory strength of XA $(1 \times .5=.5)$ and one third of the inhibitory strength of XAB $(1 \times .33=.33)$, leaving A slightly positive if the asymptotic values calculated from the preceding paragraph are used [Trial $\mathrm{A}=(.5) \mathrm{XA}+$ $(.33) \mathrm{XAB}=(.5) 2.77+(.33)-3.69=.17 \lambda]$. The same prediction follows for $\mathrm{B}$, which plays the same stimulus role as $\mathrm{A}$. If $\mathrm{A}$ and $\mathrm{B}$ were subsequently reinforced, they should then each gain $.83 \lambda$ in strength, bringing responding from $.17 \lambda$ to $1 \lambda$. However, this treatment would not undermine the original discrimination. One third of the strength gained by each of A and B would generalize to $\mathrm{XAB}$, whereas one half of the strength gained by A would generalize to XA and one half of the strength gained by $\mathrm{B}$ would generalize to XB. This state of affairs would result in almost perfect retention of the original discrimination. Of course, one inspirational force behind the development of configural theory was an attempt to explain such resistance to interference (e.g., Pearce \& Wilson, 1991).

This last advantage of configural theory would seem to close the issue. However, recent theory and research have suggested that a purely elemental learning might yet explain negative patterning. Williams et al. (2004) introduced a variant of the Rescorla-Wagner (1972) model, based on the principles embodied in the real-time connectionist account of McLaren and Mackintosh (2002). According to this account, individual CSs evoke partially overlapping sets of elements, making the pattern of activation on compound trials different from merely the sum of the separate contributions of the individual elements. In an $\mathrm{XA}+, \mathrm{XB}+, \mathrm{XAB}-$ discrimination, the stimuli $\mathrm{X}$, $\mathrm{A}$, and $\mathrm{B}$ are thought to have a shared ability to activate common elements in a nonadditive fashion, as well as an ability to evoke core elements that are not activated by any other stimulus. For example, when $\mathrm{X}$ is presented, it is thought to excite common elements that it shares with $\mathrm{A}$ and $\mathrm{B}$, as well as core elements that are nonshared. This idea is implemented by assuming that $\mathrm{X}, \mathrm{A}$, and $\mathrm{B}$ consist of $X+x a+x b, A+x a+a b$, and $B+x b+a b$, respectively, where the uppercase letters represent core elements and the lowercase bigrams represent common elements that can be activated by the presence of any one 
of the two stimuli denoted. It is further assumed that common elements are not twice activated if both stimuli are simultaneously present (e.g., the xa common elements are activated once on XA trials when both X and A are present). Applied to the discrimination at hand, this gives us

$$
\begin{aligned}
& \text { Trial XA: } V_{\mathrm{X}}+V_{\mathrm{A}}+V_{\mathrm{xa}}+V_{\mathrm{xb}}+V_{\mathrm{ab}}=1 \lambda, \\
& \text { Trial XB: } V_{\mathrm{X}}+V_{\mathrm{B}}+V_{\mathrm{xa}}+V_{\mathrm{xb}}+V_{\mathrm{ab}}=1 \lambda,
\end{aligned}
$$

and

$$
\text { Trial XAB: } V_{\mathrm{X}}+V_{\mathrm{A}}+V_{\mathrm{B}}+V_{\mathrm{xa}}+V_{\mathrm{xb}}+V_{\mathrm{ab}}=0 \lambda \text {. }
$$

Note, in this instance, that the common elements are activated on all the trials, because at least one of the two stimuli needed is always present. It should also be apparent that reinforced trials differ from unreinforced trials only in the absence of the activation of a single core element. That is, the otherwise activated core element of B is not activated on $\mathrm{XA}+$ trials, and the otherwise activated core element of $\mathrm{A}$ is not activated on $\mathrm{XB}+$ trials. With equality of salience, formal simulation of this problem reveals the following asymptotic values: $V_{\mathrm{X}}=.5 \lambda, V_{\mathrm{A}}=-1 \lambda$, $V_{\mathrm{B}}=-1 \lambda, V_{\mathrm{xa}}=.5 \lambda, V_{\mathrm{xb}}=.5 \lambda$, and $V_{\mathrm{ab}}=.5 \lambda$. Thus, the discrimination is solved. In addition, $\mathrm{X}$ is correctly predicted to demonstrate superexcitatory properties in test (i.e., $\mathrm{X}=V_{\mathrm{X}}+V_{\mathrm{xa}}+V_{\mathrm{xb}}=1.5 \lambda$ ). The theory is properly regarded as elemental because it is a descendent of the Rescorla-Wagner model and does not invoke any special internal representations for combinations of elements. On the other hand, the theory acts as if it were decidedly configural, in the sense that stimulus compounds are not processed as merely the algebraic sum of their constituent parts but, rather, as different patterns of activation.

This last point allows us to understand why this simple unvarnished version of the McLaren-Mackintosh theory without real-time learning, hereafter referred to as the overlapping elements model, correctly allows for retention of a previously learned $\mathrm{XA}+, \mathrm{XB}+, \mathrm{XAB}-$ discrimination after the $\mathrm{A}$ and $\mathrm{B}$ components are rendered excitatory. When A and B are reinforced after complex negative patterning, the theory leads to the expectation that any increases in associative strength will be widely distributed among the core elements of $\mathrm{A}$ and $\mathrm{B}$ and the shared elements that each of them activates: $V_{\mathrm{xa}}, V_{\mathrm{xb}}$, and, in particular, $V_{\mathrm{ab}}$, which is activated on both $\mathrm{A}+$ and $\mathrm{B}+$ trials. Because the majority of these elements are activated on the three trial types of the original discrimination, it follows that responding will be enhanced generally in test. When the asymptotic values in the preceding paragraph are used, responding $\left(V_{\mathrm{all}}\right)$ to $\mathrm{XA}$ and to $\mathrm{XB}(2.25 \lambda)$ is

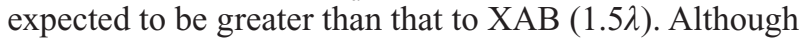
the difference between reinforced and unreinforced trials decreases from $1 \lambda$ to $.75 \lambda$, it is certainly not reversed, as would be anticipated according to the Rescorla-Wagner (1972) theory. Thus, this particular feedforward network with elemental representation has the potential to provide a general account of resistance to interference. Catastrophic interference (McCloskey \& Cohen, 1989), the loss of old learning in the face of new learning, is not a necessary property of elemental representation in singlelayer network, as it is sometimes assumed to be (e.g., Shanks, Darby, \& Charles, 1998).

Under high learning rates, this elegantly simple model also correctly predicts that an irrelevant stimulus may slow acquisition of a complex negative patterning discrimination, as does the theory of Pearce (1987), distinguishing it further from the Rescorla-Wagner (1972) model (for a discussion, see McLaren \& Mackintosh, 2002). By contrast, the theory predicts facilitated acquisition, as does the Rescorla-Wagner model, if learning rates are low, which might explain some mixed results in pigeon autoshaping and rabbit eyelid conditioning in otherwise identical experiments. Redhead and Pearce (1995) reported that two-element compounds are more difficult to discriminate from the three-element compound from which they are derived than are single elements. In their experiments, pigeons acquired an $\mathrm{A}+, \mathrm{B}+, \mathrm{X}+, \mathrm{AB}+, \mathrm{XA}+, \mathrm{XB}+$, $\mathrm{XAB}-$ discrimination. The $\mathrm{CSs}$ for the pigeons were color dots on a television screen behind a response key. As the discrimination developed, the single stimuli $(\mathrm{A}+$, $\mathrm{B}+$, and $\mathrm{X}+$ ) were easier to discriminate from the unreinforced $\mathrm{XAB}-$ than were the compounds $\mathrm{XA}+, \mathrm{XB}+$, and $\mathrm{AB}+$. In an otherwise similar set of experiments in rabbit eyelid conditioning, Myers, Vogel, Shin, and Wagner (2001) found results consistent with the predictions of the Rescorla-Wagner theory. They employed a tone, a light, and a vibration to the chest as CSs and a corneal airpuff as the US. Although the basis for the difference is unknown (Pearce \& George, 2002), the mixed results found in pigeon autoshaping and rabbit eyelid conditioning might be caused by different learning rates. According to the overlapping elements model (for a complete derivation, see McLaren \& Mackintosh, 2002), high rates of learning are expected to favor a result consistent with the configural theory of Pearce (1987), and low rates of learning are expected to favor a result consistent with the Rescorla-Wagner theory.

The present experiments were performed to examine whether either the configural theory of Pearce (1987) or the overlapping elements model correctly predicts patterns of interference after complex negative patterning in an appetitive conditioning preparation with rats. Retention of an $\mathrm{XA}+, \mathrm{XB}+, \mathrm{XAB}-$ discrimination was examined after reinforcement of (1) the A and B elements, (2) the $\mathrm{AB}$ compound, or (3) the XAB triplet. In the retention test, the rats were returned to the original discrimination in the absence of the US, to determine whether responding to XA and $\mathrm{XB}$ would continue to be greater than that to $\mathrm{XAB}$.

In this situation, the two theories make quite different predictions about the experimental outcome. The theory of Pearce (1987) predicts that interference will depend on the relative similarity of the stimulus complexes used in acquisition and interference. Interference is expected when the reinforced stimulus complex in the interference stage is relatively more similar to the originally unreinforced XAB triplet of acquisition than to the originally reinforced XA and $\mathrm{XB}$ stimulus complexes. Of the three 
interference treatments examined, the $\mathrm{AB}$ treatment is expected to produce the most interference, because the reinforced $\mathrm{AB}$ compound bears a high similarity to $\mathrm{XAB}$ (.67), and a much lower similarity to each of XA and $\mathrm{XB}$ (.25). Substantial interference is also expected when the $\mathrm{XAB}$ triplet is itself reinforced after acquisition. Reinforcement of $\mathrm{XAB}$ is expected to produce a larger increase in responding to $\mathrm{XAB}$ (similarity $=1$ ) than to each of $\mathrm{XA}$ and $\mathrm{XB}$ (similarity $=.67$ ). As was described earlier, minimal interference is expected when both A and B are reinforced on separate trials. Neither stimulus is more similar to XAB (.33) than to either XA (.50) or XB (.50).

By contrast, the predictions of the overlapping elements model vary as a function of the relative salience of the core and common elements. Under equality of salience, the theory predicts minimal interference under all conditions. As was derived earlier, reinforcement of $\mathrm{A}$ and $\mathrm{B}$ is expected to have only a minor detrimental effect on the original discrimination $(\mathrm{XA}=\mathrm{XB}=2.25 \lambda$, $\mathrm{XAB}=1.5 \lambda$ ), which is consistent with the results of Williams et al. (2002). Similar low levels of interference are also expected after reinforcement of the $\mathrm{AB}$ compound. During the interference stage, the excitatory associative strength available through reinforcement of the $\mathrm{AB}$ compound is distributed five ways among the core elements of $\mathrm{A}$ and $\mathrm{B}$ and the common elements $\mathrm{ab}, \mathrm{ax}$, and bx. In test, responding to $\mathrm{XA}$ or $\mathrm{XB}$ is expected to continue to exceed that evoked by $\mathrm{XAB}(\mathrm{XA}=\mathrm{XB}=2.2 \lambda, \mathrm{XAB}=1.5 \lambda)$. Perhaps the most striking prediction of the model is that reinforcement of XAB itself is expected to produce the least interference. Because there is a six-way distribution of associative strength across representations, the original discrimination is well retained $(\mathrm{XA}=\mathrm{XB}=1.83 \lambda$, $\mathrm{XAB}=1.0 \lambda$ ).

Of course, it would not be surprising if the relative salience of the core and common elements differed from each other and/or among themselves. To understand the predictions of the overlapping elements model, it is first necessary to appreciate that variations in rate learning parameters have no effect on the terminal strength of the A and $\mathrm{B}$ core elements, which always approach $-1 \lambda$. This occurs because the only difference between the reinforced and the unreinforced trials is the absence of a single core element. Given this, the core element X and the common elements $\mathrm{xa}, \mathrm{xb}$, and ab must collectively share an associative strength of $2 \lambda$, permitting $V_{\text {all }}$ to approach $1 \lambda$ on reinforced trials and $0 \lambda$ on unreinforced trials. Because $\mathrm{X}$ and the common elements occur together in acquisition and test, it becomes a moot point as to which has the most strength. Only if the core elements of A or B were to gain excessive amounts of excitatory strength would there be a proportionately greater increase in responding to $\mathrm{XAB}$ than to XA and XB (interference). It follows that separate reinforcement of the $\mathrm{A}$ and $\mathrm{B}$ elements or reinforcement of the $\mathrm{AB}$ compound might produce a meaningful level of interference if the core elements of A and B were relatively more salient than their common elements. Regardless of salience, the overlapping elements model predicts that reinforcement of XAB will always produce less interference (usually substantially less) than will reinforcement of the $\mathrm{A}$ and $\mathrm{B}$ elements or the $\mathrm{AB}$ compound.

Experiments 1 and 2 established the degree of interference caused by $\mathrm{AB}+$ and $\mathrm{XAB}+$ interference treatments, respectively, relative to two types of controls. There are no prior reports on the ability of these treatments to undermine retention of an $\mathrm{XA}+, \mathrm{XB}+, \mathrm{XAB}-$ discrimination. Experiment 3 directly compared levels of interference after $\mathrm{XAB}+, \mathrm{AB}+$, and $\mathrm{A}+/ \mathrm{B}+$ interference treatments. All of the experiments used exactly the same stimuli and training conditions that had previously allowed $\mathrm{X}$ to derive superexcitatory properties after $\mathrm{XA}+, \mathrm{XB}+, \mathrm{XAB}-$ discrimination training (Williams et al., 2004; Williams et al., 2002).

\section{EXPERIMENT 1}

The goal of Experiment 1 was to establish sensitivity to interference in a theory-neutral way. Both elemental and configural theories are in agreement that the $\mathrm{AB}+$ interference procedure might interfere with retention of a complex negative patterning discrimination, and thus, this procedure was used in Experiment 1. As is shown in Table 1, Experiment 1 consisted of three groups of rats. In acquisition, all the groups received a discrimination in which food was presented after $\mathrm{Y}, \mathrm{XA}$, and $\mathrm{XB}$, but not after $\mathrm{XAB}$. Next, in the interference stage, the experimental group $(\exp A B)$ received $\mathrm{AB}+$ and $\mathrm{CD}-$ trials. The purpose of the $\mathrm{CD}$ - trials was to ensure that excitatory conditioning to $\mathrm{AB}$ was stimulus specific. Controls received one of two treatments that, in theory, should have little impact on retention of the original discrimination. The rats in the rest group simply remained in their home cages, whereas the rats in the discrimination (discrim) group learned to respond to $\mathrm{CD}+$ and not to $\mathrm{AB}-$. Thus,

Table 1

Design of Experiment 1

\begin{tabular}{|c|c|c|c|}
\hline \multirow[b]{2}{*}{ Group } & \multicolumn{3}{|c|}{ Stage } \\
\hline & Acquisition & Interference & Test \\
\hline ExpAB & $\mathrm{Y}+, \mathrm{XA}+, \mathrm{XB}+, \mathrm{XAB}-$ & $\mathrm{AB}+, \mathrm{CD}-$ & $\mathrm{XA}-, \mathrm{XB}-, \mathrm{XAB}-$ \\
\hline Discrim & $\mathrm{Y}+, \mathrm{XA}+, \mathrm{XB}+, \mathrm{XAB}-$ & $\mathrm{AB}-, \mathrm{CD}+$ & $\mathrm{XA}-, \mathrm{XB}-, \mathrm{XAB}_{-}$ \\
\hline Rest & $\mathrm{Y}+, \mathrm{XA}+, \mathrm{XB}+, \mathrm{XAB}-$ & & $\mathrm{XA}-, \mathrm{XB}^{-}, \mathrm{XAB}-$ \\
\hline \multicolumn{4}{|c|}{$\begin{array}{l}\text { Note-The stimuli in Experiment } 1 \text { were as follows: } \mathrm{X} \text { and } \mathrm{Y}=\text { high- or low- } \\
\text { pitch tone (counterbalanced), } \mathrm{A} \text { and } \mathrm{C}=\text { clicker or white noise (counterbal- } \\
\text { anced), and } \mathrm{B} \text { and } \mathrm{D}=\text { panel light or flashing houselight (counterbalanced). } \\
\text { Trials were either food reinforced ( }+ \text { ) or not reinforced }(-) \text {. }\end{array}$} \\
\hline
\end{tabular}


the exp $\mathrm{AB}$ and discrim groups differed only in whether $\mathrm{AB}$ or $\mathrm{CD}$ was reinforced or not. All three groups were then returned to the original discrimination in the absence of food reinforcement $(\mathrm{XA}-, \mathrm{XB}-, \mathrm{XAB}-)$. The question of interest was whether the $\mathrm{AB}+$ trials would selectively interfere with retention of the previously learned discrimination in the expAB group.

\section{Method}

Subjects. The subjects were 24 experimentally naive male Sprague-Dawley rats (Rattus norvegicus) from the Charles River Canada colony (St. Constant, Quebec). On arrival, the rats were 90 days old and weighed approximately $275 \mathrm{~g}$. They were housed in pairs and kept in a colony room that operated on a 16:8-h light: dark cycle starting at $0600 \mathrm{~h}$. After a 2-week adaptation period that included handling and free access to food, the rats were gradually reduced to $80 \%$ of their free-feeding weights by a food-restricted diet. They were maintained at this level for the duration of the experiment. Water was freely available in their home cages.

Apparatus. Eight identical chambers $(30.8 \times 22.0 \times 27.5 \mathrm{~cm}$; MED Associates, Georgia, VT) were enclosed in separate chests (Grason-Stadler, West Concord, MA) that attenuated light and sound. A ventilation fan provided masking noise that averaged $70 \mathrm{~dB}$. The side walls and ceiling of each chamber were made of clear Perspex, and the stimulus panel and back wall were made of aluminum. The chamber floor was made of 18 stainless steel rods, $5 \mathrm{~mm}$ in diameter, spaced $11 \mathrm{~mm}$ apart. The steel rods ran parallel to the stimulus panel. Located in the center of the stimulus panel was a $5.0 \times 5.0 \mathrm{~cm}$ aperture, $2.0 \mathrm{~cm}$ above the grid floor. A single food pellet (45 mg; Formula 21, Bio-Serv, Frenchtown, NJ) could be delivered into a food cup at the base of the aperture. When the rat's head was in the aperture, it interrupted an infrared photobeam that was detected by the computer. A speaker mounted in the middle of the ceiling permitted the delivery of three auditory cues: a $0.5-\mathrm{kHz}$ low tone, an $8.0-\mathrm{Hz}$ clicking sound, and white noise. A Sonalert module located $1.0 \mathrm{~cm}$ to the right of the speaker allowed presentation of a $2.9-\mathrm{kHz}$ high tone. All of the auditory stimuli, $10 \mathrm{sec}$ in duration, were presented $8 \mathrm{~dB}$ above the background level of noise produced by the ventilation fan. Visual stimuli included the steady illumination of the panel light $(3 \mathrm{~W})$, situated $9.5 \mathrm{~cm}$ above the chamber floor to the right of the food aperture, and the flashing off and on of the houselight $(3 \mathrm{~W})$ in 0.5 -sec cycles, located on the back wall, $3.0 \mathrm{~cm}$ from the top of the chamber. A computer, equipped with MED-PC software (MED Associates), controlled the equipment. In all the experiments, the high tone and the low tone served the stimulus roles of $\mathrm{X}$ and $\mathrm{Y}$ (counterbalanced), the clicker and white noise served as A and C (counterbalanced), and the flashing of the normally illuminated houselight and the steady illumination of the panel light served as B and D (counterbalanced).

In all stages, head entry duration was monitored during the $10 \mathrm{sec}$ period just prior to the presentation of the CS (pre-CS period) and during the $10 \mathrm{sec}$ CS (CS period). Every $20 \mathrm{msec}$, the computer read inputs from the head entry detectors to determine whether any of the beams were momentarily interrupted and, if so, incremented the appropriate duration counter(s) by $20 \mathrm{msec}$. Difference scores were calculated to determine the extent to which responding during the CS period exceeded baseline (CS period minus pre-CS period; maximum $=10 \mathrm{sec}$ ). These corrected duration scores were examined with repeated measures ANOVAs, using $p<.05$ as a rejection criterion.

Procedure. Prior to training, the rats sampled the food pellets in their home cages. They were then exposed to a single session of magazine training in the experimental chambers. Pellets were delivered in the absence of any stimuli on a variable-time 30 -sec schedule. This session lasted $30 \mathrm{~min}$.
All three experiments reported in this article used a common acquisition stage. Acquisition consisted of 40 sessions of XA+, $\mathrm{XB}+, \mathrm{XAB}-$ discrimination learning. Both $\mathrm{XA}$ and $\mathrm{XB}$ signaled the delivery of a single food pellet. Food pellets were withheld on $\mathrm{XAB}$ - trials. To aid learning, there were more presentations of the unreinforced XAB than of the reinforced XA or XB. Of the 42 trials in each session, there were 6 trials each of $\mathrm{Y}+, \mathrm{XA}+$, and $\mathrm{XB}+$ and 24 trials of $\mathrm{XAB}-$. Reinforced $\mathrm{Y}$ trials were included to maintain compatibility with prior experiments (Williams et al., 2004; Williams et al., 2002). A mean intertrial interval of $70 \mathrm{sec}$ was used throughout Experiments 1-3, including Stage 1 of this experiment.

At the end of acquisition, the rats were allocated into three groups of 8 for the interference stage. Groups were formed quasirandomly in order to match them for discrimination learning and pre-CS responding. Statistical analyses of Experiments 1-3 confirmed that pre-CS responding did not differ among the groups in any stage. Because the groups were formed by eliminating differences in pre-CS responding, for brevity, we will limit our report of pre-CS responding to the following values: Experiment 1 , acquisition $=3.59 \mathrm{sec}$, interference $=3.22 \mathrm{sec}$, and test $=2.08 \mathrm{sec}$; Experiment 2, acquisition $=2.81 \mathrm{sec}$, interference $=2.64 \mathrm{sec}$, and test $=1.43 \mathrm{sec}$; and Experiment 3, acquisition $=2.45 \mathrm{sec}$, interference $=2.62 \mathrm{sec}$, and test $=1.23 \mathrm{sec}$.

In the interference stage, the exp $\mathrm{AB}$ group received 18 trials of each $\mathrm{AB}+$ and $\mathrm{CD}-$ in each of four sessions. Trial order was randomized. The discrim group was treated similarly, except that CD, rather than $\mathrm{AB}$, was reinforced. The rats in the rest group received no interference trials and stayed in their home cages.

In test, the stimuli from acquisition were presented in the absence of the US. All the rats received a single session of 15 trials each of $\mathrm{XA}-, \mathrm{XB}-$, and $\mathrm{XAB}-$. Each block of test trials included 1 trial each of $\mathrm{XA}-, \mathrm{XB}-$, and $\mathrm{XAB}-$, presented in random order. To assess retention, we asked whether averaged responding to XA and $\mathrm{XB}$, which played the same stimulus role, was greater than that to XAB. Planned orthogonal contrasts examined (1) whether responding to the previously reinforced $\mathrm{XA}$ and $\mathrm{XB}$, averaged, exceeded that to the previously unreinforced XAB in each group (a withingroup measure of retention) and (2) whether similar levels of retention were evident in the two control groups, which differed from the experimental group (between-groups interaction contrasts; Keppel \& Zedeck, 1989).

\section{Results and Discussion}

In acquisition, all the groups gradually learned to discriminate the stimuli. A 3 (group) $\times 4$ (stimulus) $\times 8$ (five-session block) ANOVA on the data of the acquisition stage revealed main effects for stimulus $[F(3,63)=$ $19.28]$ and block $[F(7,147)=23.19]$ and an interaction of block and stimulus $[F(21,441)=1.82]$. The interaction was due to the emergence of discriminative responding to the stimuli across blocks. Responding to all the stimuli was low during the first block. By the last block, responding to the reinforced $\mathrm{Y}(3.22 \mathrm{sec}), \mathrm{XA}(2.87 \mathrm{sec})$, and $\mathrm{XB}$ $(3.41 \mathrm{sec})$ was greater than that to the unreinforced $\mathrm{XAB}$ $[1.73 \mathrm{sec} ; F(1,63)=24.63]$. Analysis of the last five-session block of acquisition confirmed there were no differences in acquisition among the groups (all $F \mathrm{~s}<1$ ).

In the interference stage, both the expAB and the discrim groups learned their respective discriminations to the same final level, although the latter group learned its discrimination more quickly (see Figure 1). A 2 (group) $\times$ 2 (reinforcement) $\times 4$ (session) ANOVA on the data of the interference stage revealed a main effect for stimulus 


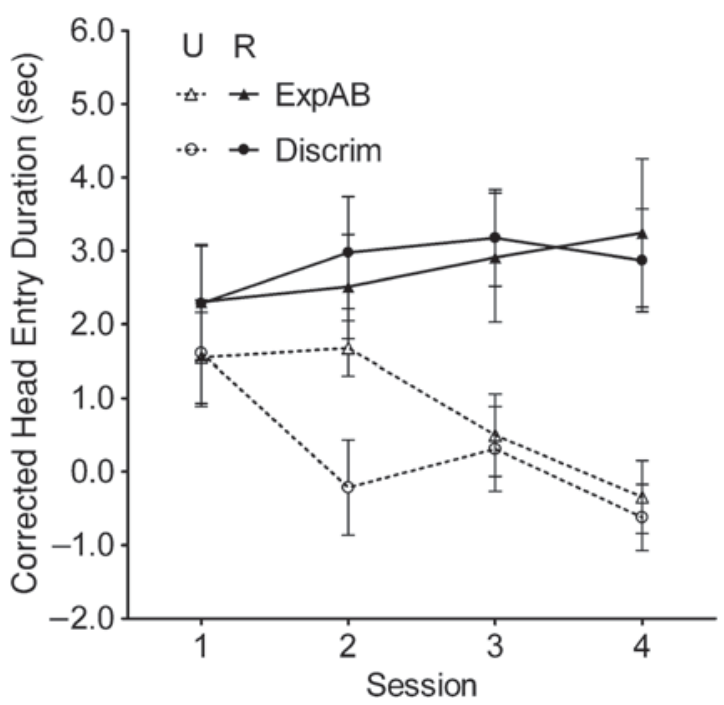

Figure 1. Mean head entry duration in seconds, corrected for pre-CS responding (CS period minus pre-CS period), during the interference stage in Experiment 1. Results are shown for the experimental (ExpAB) and discrimination (Discrim) groups as a function of session and stimulus. For the expAB group, the foodreinforced $A B$ is shown as black triangles, and the unreinforced $\mathrm{CD}$ is shown as white triangles. For the discrim group, the foodreinforced $C D$ is shown as black circles, and the unreinforced $A B$ is shown as white circles. $U=$ unreinforced and $R=$ reinforced. $A$ and $C=$ clicker or white noise (counterbalanced); $B$ and $D=$ panel light or flashing houselight (counterbalanced).

$[F(1,14)=36.86]$ and interactions of session and reinforcement $[F(3,42)=11.23]$ and of group, reinforcement, and session $[F(3,42)=3.50]$. The triple interaction confirms that differential responding to the reinforced and unreinforced stimuli first emerged in the discrim group $[$ Session $2 ; F(1,7)=37.46]$ and only thereafter in the expAB group [Session 3; $F(1,7)=14.79]$. On Session 2, the discrim group withheld responding to the unreinforced stimulus complex to a greater extent than did the expAB group. In the discrim group, this advantage was presumably due to the perceptual similarity of unreinforced $\mathrm{AB}$ of the interference stage to the previously unreinforced $\mathrm{XAB}$ of acquisition. Final levels of discriminative responding were similar in Session 4.

The major finding was that reinforcement of $\mathrm{AB}$ undermined retention of the original discrimination, as is shown in Figure 2. As expected, responding to XA and XB, averaged, was greater than that to $\mathrm{XAB}$ in the two control groups [discrim $F(1,7)=27.94$; rest $F(1,7)=16.60]$. By contrast, there was no statistical evidence that the original discrimination had been retained in the expAB group $[F(1,7)<1]$. Repeated measures ANOVA confirmed these group differences in retention by revealing a group $\times$ retention interaction $[F(2,21)=4.50]$. The control and rest groups, which showed similar levels of retention, differed from the experimental group $[F(1,21)=8.91]$.

In summary, all three groups of rats learned to discriminate the stimuli during the acquisition stage. During the interference stage, the experimental and control groups gradually learned to respond to the reinforced compound and not to the unreinforced compound. In test, reinforcement of $A B$ preferentially interfered with retention of the original discrimination. On the other hand, reinforcement of $\mathrm{CD}$ had no detectable effect when responding was compared with that of another group of rats that had rested during the interference stage. On the basis of these data, we can conclude that complex negative patterning discriminations are not always resistant to interference.

\section{EXPERIMENT 2}

Experiment 2 tested whether reinforcement of $\mathrm{XAB}$ would interfere with retention of a complex patterning discrimination. As is shown in Table 2, this experiment followed a design similar to that used in Experiment 1. Three groups of rats first acquired a $\mathrm{Y}+, \mathrm{XA}+, \mathrm{XB}+$, $\mathrm{XAB}-$ discrimination. During the interference stage, the $\operatorname{expXAB}$ group received $\mathrm{XAB}+$ and $\mathrm{XCD}-$ trials, and the discrim group received $\mathrm{XAB}-$ and $\mathrm{XCD}+$ trials. Again, the rats in the rest group remained in their home cages. In test, the three groups were returned to the original discrimination in the absence of reinforcement (XA$\mathrm{XB}-, \mathrm{XAB}-)$. The question of interest was whether reinforcement of XAB would cause interference, as would be predicted by configural theory, or would have only a minimal impact on retention of the discrimination, as would be predicted by the overlapping elements model.

\section{Method}

Subjects and Apparatus. The subjects were 24 experimentally naive male rats (Rattus norvegicus) of Sprague-Dawley descent

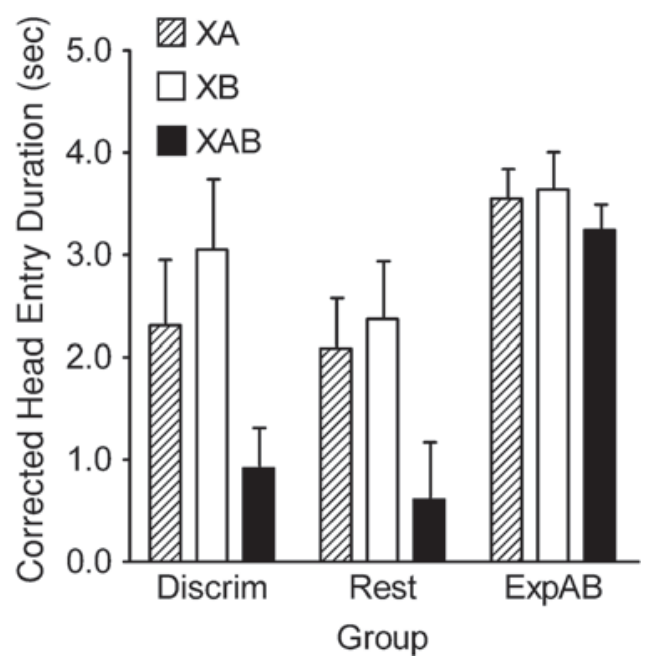

Figure 2. Mean head entry duration in seconds, corrected for pre-CS responding (CS period minus pre-CS period), during the test stage in Experiment 1. Results are shown for the discrimination (Discrim) group, a group that remained in the home cages during the prior interference stage (Rest), and the experimental $(\operatorname{ExpAB})$ group. All the groups received unreinforced presentations of the $\mathrm{XA}, \mathrm{XB}$, and $\mathrm{XAB}$ stimuli, where $\mathrm{X}=$ low or high tone (counterbalanced), $\mathrm{A}=$ clicker or white noise (counterbalanced); B = panel light or flashing houselight (counterbalanced). 
Table 2

Design of Experiment 2

\begin{tabular}{lccc}
\hline & \multicolumn{3}{c}{ Stage } \\
\cline { 2 - 4 } Group & Acquisition & Interference & Test \\
\hline ExpXAB & $\mathrm{Y}+, \mathrm{XA}+, \mathrm{XB}+, \mathrm{XAB}-$ & $\mathrm{XAB}+, \mathrm{XCD}-$ & $\mathrm{XA}-, \mathrm{XB}-, \mathrm{XAB}-$ \\
Discrim & $\mathrm{Y}+, \mathrm{XA}+, \mathrm{XB}+, \mathrm{XAB}-$ & $\mathrm{XAB}-, \mathrm{XCD}+$ & $\mathrm{XA}-, \mathrm{XB}-, \mathrm{XAB}-$ \\
Rest & $\mathrm{Y}+, \mathrm{XA}+, \mathrm{XB}+, \mathrm{XAB}-$ & & $\mathrm{XA}-, \mathrm{XB}-, \mathrm{XAB}-$ \\
\hline
\end{tabular}

Note-The stimuli in Experiment 2 were as follows: $X$ and $Y=$ high- or low-pitch tone (counterbalanced), $\mathrm{A}$ and $\mathrm{C}=$ clicker or white noise (counterbalanced), and $\mathrm{B}$ and $\mathrm{D}=$ panel light or flashing houselight (counterbalanced). Trials were either food reinforced $(+)$ or not reinforced $(-)$.

(Charles River Canada, St. Constant, Quebec). They were housed and maintained in the same fashion as that in Experiment 1. The apparatus was also the same.

Procedure. The procedures used in Experiment 2 differed from those in Experiment 1 only in the interference stage. The acquisition and test stages were identical to those in Experiment 1. In the interference stage, the $\operatorname{expXAB}$ group was presented with the stimulus complexes of $\mathrm{XAB}+$ and $\mathrm{XCD}-$, whereas the discrim group was presented with $\mathrm{XAB}-$ and $\mathrm{XCD}+$. Again, the rats in the rest group remained in their home cages during the interference stage.

\section{Results and Discussion}

As was expected, the groups learned to discriminate the stimuli over the course of acquisition. A 3 (group) $\times 4$ (stimulus) $\times 8$ (five-session block) ANOVA on these data revealed main effects for stimulus $[F(3,63)=17.30]$ and block $[F(7,147)=4.32]$ and an interaction of block and stimulus $[F(21,441)=3.17]$. Further examination of the interaction showed that responding to all the stimuli was similar and low during the first block. By the last block of the acquisition stage, however, the stimuli were discriminated $[F(3,63)=12.17]$. As expected, responding was higher to the reinforced $\mathrm{Y}(3.10 \mathrm{sec}), \mathrm{XA}(3.16 \mathrm{sec})$, and $\mathrm{XB}(2.77 \mathrm{sec})$ than to the unreinforced XAB [1.86 sec; $F(1,63)=39.40]$. No group differences in discriminative responding were found during the last five-session block of acquisition (all $F \mathrm{~s}<1$ ).

During the interference stage, the rats learned to respond preferentially to the reinforced compound. A 2 (group) $\times$ 2 (reinforcement) $\times 4$ (session) ANOVA revealed main effects for reinforcement $[F(1,14)=36.86]$ and an interaction of reinforcement and session $[F(3,42)=9.87]$. As can be seen in Figure 3, there was a tendency toward faster acquisition in the discrim group than in the expXAB group. Unlike in Experiment 1, this numerical difference was unreliable and did not produce an interaction of group, reinforcement, and session $[F(3,42)=2.12, p<.12]$.

The data of greatest interest are those from the test stage (see Figure 4). The main finding was that reinforcement of XAB had a detrimental impact on retention of the original discrimination. As expected, responding to XA and $\mathrm{XB}$, averaged, was greater than that to $\mathrm{XAB}$ in the discrim $[F(1,7)=15.77]$ and rest $[F(1,7)=53.71]$ groups, which did not differ from each other. By contrast, there was no evidence in the expXAB group that the previously learned discrimination had been retained $[F(1,7)<1]$. A repeated measures ANOVA confirmed these group differences in retention by revealing a group $\times$ retention interaction
$[F(2,21)=5.45]$. A direct comparison confirmed that the pattern of responding for the expXAB group was different than that for the rest and control groups $[F(1,21)=$ 14.12] which, unlike the expXAB group, had retained the discrimination. Thus, contrary to the predictions of the overlapping elements model, Experiment 2 showed a substantial degree of interference after reinforcement of XAB.

\section{EXPERIMENT 3}

The purpose of Experiment 3 was to directly compare levels of interference in a single experiment. As is shown in Table 3, this experiment consisted of a rest group and three experimental groups (expA/B, expAB, and expXAB). During the acquisition stage, all the groups learned the

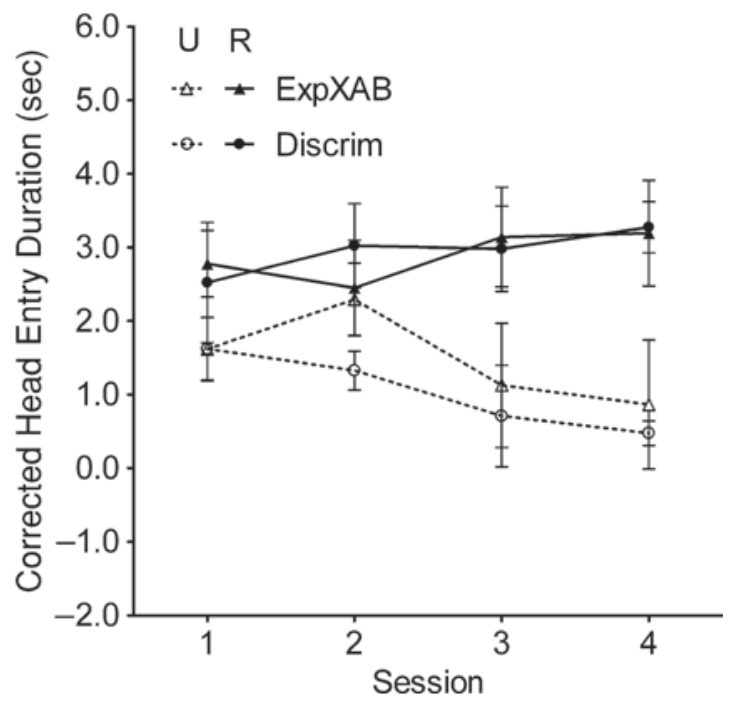

Figure 3. Mean head entry duration in seconds, corrected for pre-CS responding (CS period minus pre-CS period), during the interference stage in Experiment 2. Results are shown for the experimental (ExpXAB) and discrim (Discrim) groups as a function of session and stimulus. For the $\operatorname{expXAB}$ group, the foodreinforced $\mathrm{XAB}$ is shown as black triangles, and the unreinforced $\mathrm{XCD}$ is shown as white triangles. For the discrim group. the foodreinforced XCD stimulus compound is shown as black circles, and the unreinforced $X A B$ is shown as white circles. $U=$ unreinforced and $R=$ reinforced. $X=$ low or high tone (counterbalanced); $A$ and $\mathrm{C}=$ clicker or white noise (counterbalanced); $\mathrm{B}$ and $\mathrm{D}=$ panel light or flashing houselight (counterbalanced). 


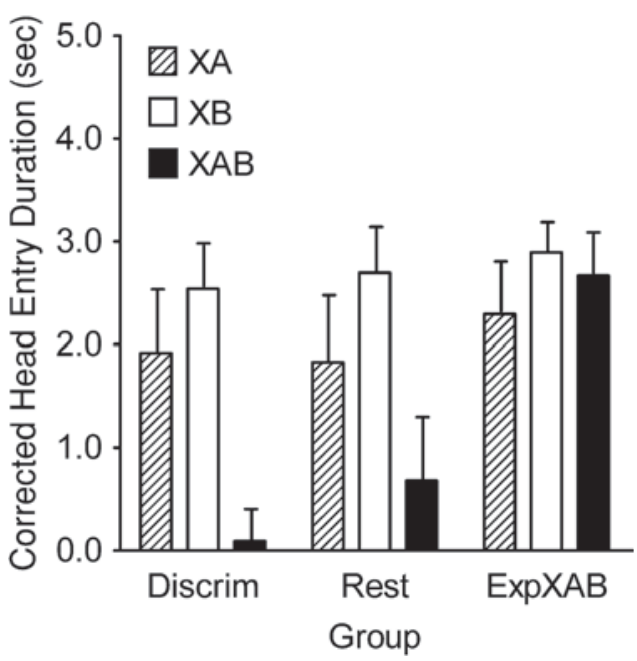

Figure 4. Mean head entry duration in seconds, corrected for pre-CS responding (CS period minus pre-CS period), during the test stage in Experiment 2. Results are shown for the discrim (Discrim) group, a group that remained in the home cages during the prior interference stage (Rest), and the experimental (ExpXAB) group. All the groups received unreinforced presentations of the $\mathrm{XA}, \mathrm{XB}$, and $\mathrm{XAB}$ stimuli. $\mathrm{X}=$ low or high tone (counterbalanced); $\mathbf{A}=$ clicker or white noise (counterbalanced); $\mathbf{B}=$ flashing houselight or panel light (counterbalanced).

$\mathrm{Y}+, \mathrm{XA}+, \mathrm{XB}+, \mathrm{XAB}-$ discrimination, just as in Experiments 1 and 2. Next, the expA/B group received $\mathrm{A}+$, $\mathrm{B}+$, and $\mathrm{CD}-$ trials, the expAB group received $\mathrm{AB}+$ and $\mathrm{CD}-$ trials, and the expXAB group received $\mathrm{XAB}+$ and $\mathrm{CD}$ - trials. Again, the rats in the rest group received no interference trials and stayed in their home cages. During the test stage, all four groups received unreinforced trials on the original discrimination.

\section{Method}

Subjects and Apparatus. The subjects were 24 experimentally naive male rats (Rattus norvegicus) of Sprague-Dawley descent (Charles River Canada, St. Constant, Quebec). They were housed and maintained in the same fashion as that in Experiment 1. The apparatus was the same as that described in Experiment 1.

Procedure. The acquisition and test stages in the present experiment were identical to those in Experiments 1 and 2. In the interference stage, the procedures used for the $\operatorname{expXAB}$, $\exp \mathrm{AB}$, and rest groups were identical to those used for comparable groups in
Experiments 1 and 2 . The expA/B group received 18 trials each of $\mathrm{A}+, \mathrm{B}+$, and $\mathrm{CD}-$.

\section{Results and Discussion}

All the groups learned to discriminate the reinforced and unreinforced stimuli in acquisition. A 4 (group) $\times 4$ (stimulus) $\times 8$ (five-session block) ANOVA on the data from the acquisition stage revealed main effects for stimulus $[F(3,84)=12.03]$ and block $[F(7,147)=4.87]$ and an interaction of block and stimulus $[F(21,441)=3.58]$. Responding to all the stimuli was low during the first block and increased in subsequent blocks in a similar manner for all the stimuli except the unreinforced $\mathrm{XAB}$, which first increased and then decreased. By the last block of acquisition, responding to $\mathrm{Y}(3.07 \mathrm{sec}), \mathrm{XA}(3.02 \mathrm{sec})$, and XB $(3.22 \mathrm{sec})$ was higher than that to XAB [1.78 sec; $F(1,84)=45.65]$. There were no differences in responding between the groups in any of the analyses.

Figure 5 shows the course of interference across sessions. In the figure, responding to the reinforced $\mathrm{A}$ and $\mathrm{B}$ in the expA/B group is averaged in order to allow comparisons with the remaining groups that had a single reinforced stimulus. As expected, differential responding to the reinforced and unreinforced stimuli emerged over sessions. A 3 (group) $\times 2$ (reinforcement) $\times 4$ (session) ANOVA revealed a main effect of stimulus $[F(1,21)=$ 106.77] and interactions of session and reinforcement $[F(3,63)=32.20]$ and of group, reinforcement, and session $[F(6,63)=4.07]$. The interaction of session and reinforcement was due to increasing differences in responding to the reinforced and the unreinforced stimuli across sessions. This difference emerged in Session $1[F(1,21)=$ 18.69] and increased in magnitude across groups until Session 4. In Session 4, differential responding was somewhat greater in the expA/B group than in the remaining groups $[F(1,21)=11.36]$, which caused the three-way interaction.

In test, there was preferential retention of the patterning discrimination only after reinforcement of A and B (see Figure 6). We first determined whether retention occurred in the rest group. As expected, the rats in the rest group retained their discrimination after 4 days of remaining in their home cages $[F(1,7)=36.20]$. On the basis of prior data, we also expected retention in the expA/B group. Consistent with prior results (Williams et al., 2002), the

Table 3

Design of Experiment 3

\begin{tabular}{lllc}
\hline & \multicolumn{3}{c}{ Stage } \\
\cline { 2 - 4 } Group & \multicolumn{1}{c}{ Acquisition } & Interference & \multicolumn{1}{c}{ Test } \\
\hline ExpA/B & $\mathrm{Y}+, \mathrm{XA}+, \mathrm{XB}+, \mathrm{XAB}-$ & $\mathrm{A}+, \mathrm{B}+, \mathrm{CD}-$ & $\mathrm{XA}-, \mathrm{XB}-, \mathrm{XAB}-$ \\
ExpAB & $\mathrm{Y}+, \mathrm{XA}+, \mathrm{XB}+, \mathrm{XAB}-$ & $\mathrm{AB}+, \mathrm{CD}-$ & $\mathrm{XA}-, \mathrm{XB}-, \mathrm{XAB}-$ \\
ExpXAB & $\mathrm{Y}+, \mathrm{XA}+, \mathrm{XB}+, \mathrm{XAB}-$ & $\mathrm{XAB}+, \mathrm{CD}-$ & $\mathrm{XA}-, \mathrm{XB}-, \mathrm{XAB}-$ \\
Rest & $\mathrm{Y}+, \mathrm{XA}+, \mathrm{XB}+, \mathrm{XAB}-$ & & $\mathrm{XA}-, \mathrm{XB}-, \mathrm{XAB}-$ \\
\hline
\end{tabular}

Note-The stimuli in Experiment 3 were as follows: $X$ and $Y=$ high- or low-pitch tone (counterbalanced), $\mathrm{A}$ and $\mathrm{C}=$ clicker or white noise (counterbalanced), and $\mathrm{B}$ and $\mathrm{D}=$ panel light or flashing houselight (counterbalanced). Trials were either food reinforced $(+)$ or not reinforced $(-)$. 


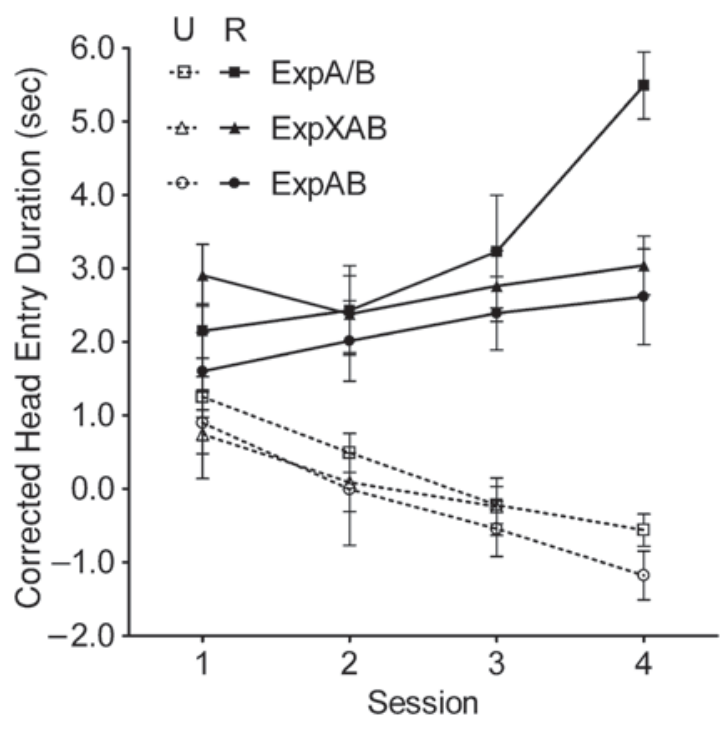

Figure 5. Mean head entry duration in seconds, corrected for pre-CS responding (CS period minus pre-CS period), during the interference stage in Experiment 3. Results are shown for the experimental groups $(\operatorname{Exp} A / B, \operatorname{ExpXAB}$, and $\operatorname{Exp} A B)$ as a function of session and stimulus. For the $\operatorname{Exp} A / B$ group, reinforced presentations of $A$ and $B$ are shown as black squares, and the unrein-

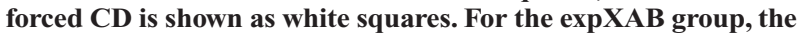
reinforced $\mathrm{XAB}$ is shown as black triangles, and the unreinforced $C D$ is shown as white triangles. For the $\exp A B$ group, reinforced presentations of $\mathrm{AB}$ are shown as black circles, and the unreinforced $C D$ is shown as white circles. $U=$ unreinforced and $R=$ reinforced. $\mathrm{X}=$ low or high tone (counterbalanced); $\mathrm{A}$ and $\mathrm{C}=$ clicker or white noise (counterbalanced); $B$ and $D=$ panel light or flashing houselight (counterbalanced).

original discrimination between the reinforced $\mathrm{XA}$ and $\mathrm{XB}$, averaged, and the unreinforced $\mathrm{XAB}$ was retained $[F(1,7)=14.25]$, even though $\mathrm{A}$ and $\mathrm{B}$ were excitatory at the end of the interference stage. On the other hand, the rats in the expAB group and, to a numerically lesser extent, the $\operatorname{expXAB}$ group showed less evidence of retention of the original discrimination. In test, differential responding to the formerly reinforced and unreinforced stimulus complexes was absent in the exp $\mathrm{AB}$ group $[F(1,7)=1.54]$, but not in the expXAB group $[F(1,7)=$ 7.04]. A repeated measures ANOVA confirmed these group differences in retention by revealing a group $\times$ retention interaction $[F(3,28)=3.21]$. As expected, there were no differences in retention between the $\operatorname{expA} / \mathrm{B}$ and the rest groups $[F(1,28)<1]$, which suggests that prior learning was unaffected by the change in the associative status of A and B. Although the data suggest that interference might be slightly stronger after reinforcement of $\mathrm{AB}$ than after that of $\mathrm{XAB}$, as is predicted by the theory of Pearce (1987, 1994), a direct comparison of the magnitude of interference in the expAB and expXAB groups did not reach significance. Taken together, the expAB and expXAB groups showed less retention than did their $\operatorname{expA} / \mathrm{B}$ and rest counterparts $[F(1,28)=8.51] .{ }^{1}$

In conclusion, there was substantial interference after reinforcement of $\mathrm{AB}$ and $\mathrm{XAB}$, as in Experiments 1 and
2, but not after reinforcement of A and B. This result is especially surprising given that final levels of excitatory conditioning were stronger in the $\operatorname{expA} / \mathrm{B}$ group than in the exp $\mathrm{AB}$ or expXAB groups. All other factors being equal, it seems reasonable to suppose that interference should increase, not decrease, with the level of excitatory conditioning obtained in the interference stage. From the perspective of configural theory, the expA/B group might be regarded as an especially appropriate control group, rather than as an experimental group. Note that the expA/B and $\exp \mathrm{AB}$ groups were equated for the total number of reinforced presentations of the $\mathrm{A}$ and $\mathrm{B}$ components, as well as for exposure to the unreinforced $\mathrm{CD}$, but only the former group retained the discrimination.

\section{GENERAL DISCUSSION}

Our results suggest that stimulus combinations acquire control over discriminative responding in complex negative patterning. Reinforcement of either AB (Experiments 1 and 3 ) or XAB (Experiments 2 and 3 ) interfered with retention of a previously learned $\mathrm{XA}+, \mathrm{XB}+, \mathrm{XAB}-$ discrimination, whereas reinforcement of A and B separately had no detectable effect (Experiment 3). Thus, retroactive interference was observed when excitation acquired in the interference stage generalized more to the previously unreinforced XAB than to the reinforced XA and XB. Otherwise, the original discrimination was retained.

One explanation for the results of Experiments 1-3 is that special representations exist for entire stimulus complexes as single units (Pearce, 1987). On this account, reinforcement of $\mathrm{XAB}$ and, particularly, $\mathrm{AB}$ is expected to

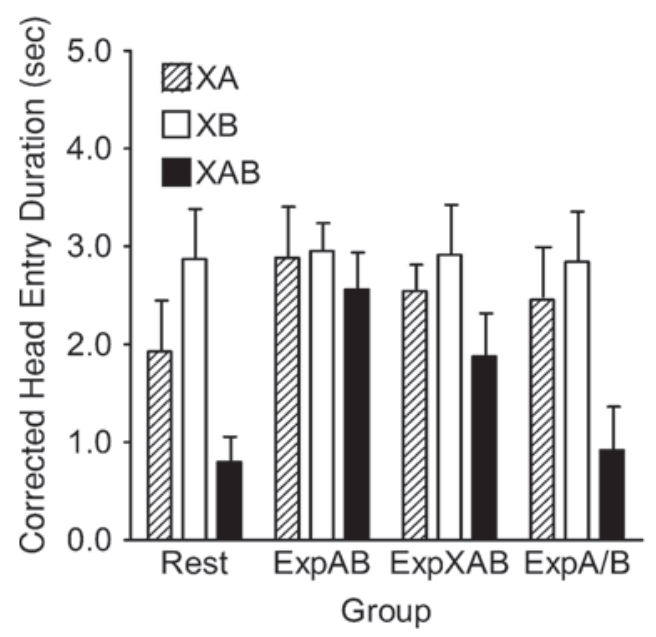

Figure 6. Mean head entry duration in seconds, corrected for pre-CS responding (CS period minus pre-CS period), during the test stage in Experiment 3. Results are shown for a group that remained in the home cages during the prior interference stage (Rest) and for the three experimental groups (ExpAB, ExpXAB, and $\operatorname{ExpA} / B$ ). All the groups received unreinforced presentations of the XA, XB, and XAB stimuli. $X=$ low or high tone (counterbalanced); $\mathbf{A}=$ clicker or white noise (counterbalanced); $B=$ flashing houselight or panel light (counterbalanced). 
increase responding to the previously unreinforced $\mathrm{XAB}$, without producing a commensurate increase in responding to XA and XB. On the other hand, separate reinforcement of $\mathrm{A}$ and $\mathrm{B}$ is predicted to enhance responding in general and to fail to undermine the original discrimination. Under equality of salience, the A and B stimuli constitute one half of the XA and XB stimulus complexes, respectively, and one third of the XAB stimulus complex. Thus, there is little basis on which to predict retroactive interference after separate reinforcement of A and B. It could be argued that a substantially greater number of reinforced $A$ and $\mathrm{B}$ trials in the interference stage might have produced some interference in the present experiments (e.g., Denniston, Chang, \& Miller, 2003; Tamai \& Nakajima, 2000). Given this, it would be prudent to withhold judgment on the question of whether complex negative patterning discriminations are completely resistant to inference after excitatory conditioning of $\mathrm{A}$ and $\mathrm{B}$.

It is natural to ask whether configural theory also finds support in the data for the interference stage itself. As was just mentioned, configural theory makes the general prediction that interference at test should be most pronounced when the reinforced stimulus complex of the interference stage is relatively more similar to the XAB unreinforced stimulus complex of acquisition than to the reinforced XA and XB stimulus complexes. It also follows that discriminative responding in the interference stage itself should be affected. The very same treatments that produce interference at test would themselves be subject to interference. In keeping with this prediction, final levels of excitatory conditioning in the interference stage were weaker in the $\exp \mathrm{AB}$ and $\operatorname{expXAB}$ groups than in the expA/B group in Experiment 3. One blemish to the record of successful predictions of configural theory is the failure to find slower discrimination learning in the expXAB group than in the discrim group in the interference stage of Experiment 2, although a similar prediction finds support in Experiment 1 .

Of particular interest is the failure of the purely elemental models considered in the introduction to this article to account for the pattern of interference observed. According to the Rescorla-Wagner (1972) theory, reinforcement of A and B should have reversed the discrimination. Because all of the components were excitatory, their associative strengths should have summated, and responding to $\mathrm{XAB}$ should have been greater than that to XA and $\mathrm{XB}$. No such interference was observed, and only reinforcement of $\mathrm{AB}$ or $\mathrm{XAB}$ had an observable impact on retention of the previously learned discrimination. These data suggest that complex negative patterning discriminations are not open to interference under the conditions specified by the Rescorla-Wagner theory.

Likewise, we found little support for a modification of the Rescorla-Wagner (1972) model in which the elements support overlapping patterns of activation. In addition to activating representations for the core features of stimuli, it might be assumed that stimuli also have the ability to activate shared representations. This model, inspired by the real-time model of McLaren and Mackintosh (2002), assumes that Stimulus A, for example, excites shared representations also activated by Stimuli B (ab) and X (ax), in addition to a nonshared representation that encodes features unique to A. Applied to the present experiments, the overlapping elements model provides a rather poor fit to the data, although it does accurately predict a number of prior results in complex negative patterning experiments (Williams et al., 2004; Williams et al., 2002). Contrary to the predictions, we found that reinforcement of $\mathrm{A}$ and $B$ was not a particularly effective means for producing interference, whereas reinforcement of XAB did affect retention of the discrimination. It is unclear to us what further modifications to the overlapping elements model might allow for an accurate prediction of the results of Experiment 3 without undoing other successful predictions. Clearly, it would be unwise to abandon this approach entirely on the basis of a single empirical finding, especially when this simple feedforward network provides a potential avenue for solving the vexing problem of catastrophic interference (French, 1991; McCloskey \& Cohen, 1989).

One advantage of the theoretical comparisons made here is the common ground provided by the adoption of a $\lambda-V$ error-correction mechanism within a simple feedforward network, making it possible to see what assumptions of the various theories are necessary to explain the results. Despite this, one might ask whether introducing a real-time component to the overlapping elements model, instead of applying the Rescorla-Wagner equation, would lead to a correct prediction. Real-time learning, such as the delta rule, allows such a model to explain such phenomena as one-trial overshadowing (McLaren \& Mackintosh, 2000) and the course of acquisition in complex negative patterning (Williams et al., 2004). Although it would be rash to dismiss such an approach, it is unclear how real-time learning would improve predictive power in this instance.

Wagner and Brandon (2001) have shown that it is possible to devise an elemental model that often makes predictions similar to those of Pearce's (1987) configural theory. On this approach, individual CSs activate internal representations, called nodes, that contain a fixed number of elements. They found that the predictions of configural theory are generally equivalent to a model in which a relatively large proportion of the nodal elements of a stimulus are inhibited in the presence of another stimulus. In the case of two stimuli, $\mathrm{X}$ and A, the presence of Stimulus X is suggested to inhibit half of the elements otherwise activated by A, and vice versa. This approach produces different representations of $\mathrm{A}$ in the presence and absence of $\mathrm{X}$ : The nodal elements of $\mathrm{A}$ are either context independent (activated regardless of X's presence) or context dependent (activated only in X's absence). Using the simulation procedures described by Pearce and George (2002), Williams et al. (2004) reported that the inhibited elements model and configural theory made different predictions about the outcome of their experiments. Thus, the two methods of representation are not identical in all cases. Applied to the present experiments, computer simulations of the inhibited elements model revealed a pattern of interference 
similar to that of the configural theory of Pearce (1987). Thus, the two are in agreement in this case, and both provide a good fit to the data.

To our knowledge, the present experiments stand as the first demonstration that relative similarity plays a role in the level of interference observed in a Pavlovian conditioning experiment. Previous demonstrations of resistance to interference (Pearce \& Wilson, 1991; Williams et al., 2002, Experiment 6) are open to the suggestion that excitatory conditioning leaves intact any inhibitory associations acquired by the stimuli during the acquisition stage (e.g., Bouton, 1993). However, if this were the only mechanism, one might expect resistance after reinforcement in all of the conditions studied in Experiments 1-3. It is especially difficult to understand why two interference procedures that had exactly the same stimulus elements in common, the $\mathrm{AB}+$ and $\mathrm{A}+\mathrm{B}+$ procedures, supported such different levels of interference (cf. Gunther, Denniston, \& Miller, 1998). Thus, the present experiments tell us something important about the mechanisms responsible for both retroactive interference and negative patterning.

\section{REFERENCES}

Bouton, M. E. (1993). Context, time, and memory retrieval in the interference paradigms of Pavlovian learning. Psychological Bulletin, 114, 80-99.

Denniston, J. C., Chang, R. C., \& Miller, R. R. (2003). Massive extinction treatment attenuates the renewal effect. Learning \& Motivation, 34, 68-86.

FrenCH, R. M. (1991). Using semi-distributed representations to overcome catastrophic forgetting in connectionist networks. In Proceedings of the 13th Annual Cognitive Science Society (pp. 173-178). Hillsdale, NJ: Erlbaum.

Gunther, L. M., Denniston, J. C., \& Miller, R. R. (1998). Conducting exposure treatment in multiple contexts can prevent relapse. Behaviour Research \& Therapy, 36, 75-91.

KeнoE, E. J. (1988). A layered network model of associative learning: Learning to learn and configuration. Psychological Review, 95, 411433.

KePpel, G., \& Zedeck, S. (1989). Data analysis for research designs: Analysis of variance and multiple regression/correlation approaches. New York: Freeman.

McCloskey, M., \& Cohen, N. J. (1989). Catastrophic interference in connectionist networks: The sequential learning problem. Psychology of Learning \& Motivation, 24, 109-165.

McLaren, I. P. L., \& Mackintosh, N. J. (2000). An elemental model of associative learning: I. Latent inhibition and perceptual learning. Animal Learning \& Behavior, 28, 211-246.

McLaren, I. P. L., \& Mackintosh, N. J. (2002). Associative learning and elemental representation: II. Generalization and discrimination. Animal Learning \& Behavior, 30, 177-200.

Myers, K. M., Vogel, E. H., Shin, J., \& Wagner, A. R. (2001). A comparison of the Rescorla-Wagner and Pearce models in a negative patterning and a summation problem. Animal Learning \& Behavior, 29, 36-45.

Pearce, J. M. (1987). A model for stimulus generalization in Pavlovian conditioning. Psychological Review, 94, 61-73.
Pearce, J. M. (1994). Similarity and discrimination: A selective review and connectionist model. Psychological Review, 101, 587-607.

Pearce, J. M. (2002). Evaluation and development of a connectionist theory of configural learning. Animal Learning \& Behavior, 30, 73-95.

Pearce, J. M., \& George, D. N. (2002). The effects of using stimuli from three different dimensions on autoshaping with a complex negative patterning discrimination. Quarterly Journal of Experimental Psychology, 55B, 349-364.

Pearce, J. M., \& Redhead, E. S. (1993). The influence of an irrelevant stimulus on two discriminations. Journal of Experimental Psychology: Animal Behavior Processes, 19, 180-190.

Pearce, J. M., \& Wilson, P. N. (1991). Failure of excitatory conditioning to extinguish the influence of a conditioned inhibitor. Journal of Experimental Psychology: Animal Behavior Processes, 17, 519-529.

Redhead, E. S., \& Pearce, J. M. (1995). Similarity and discrimination learning. Quarterly Journal of Experimental Psychology, 48B, 46-66.

ResCORLA, R. A. (1972). "Configural” conditioning in discrete-trial bar pressing. Journal of Comparative \& Physiological Psychology, 79 , 307-312.

Rescorla, R. A. (1973). Evidence for a "unique stimulus" account of configural conditioning. Journal of Comparative \& Physiological Psychology, 85, 331-338.

Rescorla, R. A., \& WAGNer, A. R. (1972). A theory of Pavlovian conditioning: Variations in the effectiveness of reinforcement and nonreinforcement. In A. H. Black \& W. F. Prokasy (Eds.), Classical conditioning II: Current research and theory (pp. 64-99). New York: Appleton-Century-Crofts.

SchmaJuK, N., \& DiCARLO, J. (1992). Stimulus configuration, classical conditioning, and hippocampal function. Psychological Review, 99, 268-305.

Shanks, D. R., Darby, R. J., \& Charles, D. (1998). Resistance to interference in human associative learning: Evidence for configural learning. Journal of Experimental Psychology: Animal Behavior Processes, 24, 136-150.

TAmai, N., \& NaKaJima, S. (2000). Renewal of formerly conditioned fear in rats after extensive extinction training. International Journal of Comparative Psychology, 13, 137-146.

WAGNER, A. R., \& BRANDON, S. E. (2001). A componential theory of Pavlovian conditioning. In R. R. Mowrer \& S. B. Klein (Eds.), Handbook of contemporary learning theory (pp. 301-336). Mahwah, NJ: Erlbaum.

Williams, D. A., Mehta, R., \& Dumont, J.-L. (2004). Conditions favoring superconditioning of irrelevant conditioned stimuli. Journal of Experimental Psychology: Animal Behavior Processes, 30, 139-148.

Williams, D. A., Mehta, R., Poworoznyk, T. M., Orihel, J. S., George, D. N., \& Pearce, J. M. (2002). Acquisition of superexcitatory properties by an irrelevant background stimulus. Journal of Experimental Psychology: Animal Behavior Processes, 28, 284-297.

Woodbury, C. B. (1943). The learning of stimulus patterns by dogs. Journal of Comparative Psychology, 35, 29-40.

\section{NOTE}

1. For the curious, the same pattern of group differences in Stage 3 is found if pairwise $t$ tests are used to determine the source of the group $X$ stimulus interactions found in Experiments 1-3. The only exception is the difference implied between the expA/B and expXAB groups in Experiment 3 , which is reliable only if a one-tailed criterion is used.

(Manuscript received September 7, 2004; revision accepted for publication February 7, 2005.) 